\title{
Risk Measurement of Futures Portfolio: An Empirical Study Based on PGARCH - EVT - Copula Model
}

\author{
Liang $\mathrm{Su}^{1}$, Lan-Ya Ma ${ }^{1}$ \\ ${ }^{1}$ Finance Department of International Business School, Jinan University, Zhuhai, China \\ Correspondence: Liang Su, Finance Department of International Business School, Jinan University, Qianshan Road \\ 206\#, Zhuhai City, Guangdong Province, Post No.519070, China.
}

Received: July 24, 2017

doi:10.11114/aef.v4i5.2548
Accepted: August 18, $2017 \quad$ Available online: August 23, 2017

URL: https://doi.org/10.11114/aef.v4i5.2548

\begin{abstract}
Financial risk management takes an important part of continuing financial globalization. From the point of financial risk management, financial risk should be controlled at the right level. Considering the characteristics of financial time series, we construct the PGARCH-EVT-Copula model that includes different aspects of statistical features in measuring the risk. With this model, we measure Value at Risk and Expected Shortfall of the futures portfolio and compare them in the risk measurement and testify the reliability with the help of Monte-Carlo simulation method. Finally, we draw a conclusion that at $95 \%$ confidence level, Expected Shortfall can better estimate the risk of assets price extreme changing. This paper provides a risk management method for stabilizing the financial market.
\end{abstract}

Keywords: risk management, PGARCH-EVT-Copula model, value at risk, expected shortfall, monte-carlo simulation

\section{Introduction}

\subsection{Background Description}

Financial globalization is an important aspect of economic globalization, and the relationship between financial markets in different countries and regions is becoming close. Especially in recent years, telecommunication technology is developing, making the financial transaction more efficient, while the risk in different markets linked to each other, exacerbates the domestic financial risks. The financial risk measurement and management are significantly necessary. Well-operating financial markets require risk management in a timely manner to adjust market incorrect trends. Empirical works show that the traditional VaR model cannot measure the fluctuation spillover effect of different markets and assets and has some limitations in today complex financial situation. In order to manage and control the financial risk, it is necessary to consider the fluctuation spillover effect in extreme cases and the potential losses that may result from them.

In order to reflect the extreme situation of market price changes, as for financial time series, we use the Extreme Value Theory (EVT) to analyze the extreme value and the upper and lower tail distributions of the yield residual series, fitting them based on the General Pareto Distribution. PGARCH model is an asymmetric GARCH model and the improved PGARCH model can be used to reflect the asymmetric distribution of financial time series. Base on the above model, we analyze the influence of the fluctuation spillover effect with the help of Copula function to connect the marginal distributions of a single asset and measure the VaR and ES, providing a means to measure and manage the financial risk.

\subsection{Research Design}

Although domestic and foreign scholars have studied many approaches to measure the risk, we aim to cover the blank of futures risk measurement and analyze the characteristic of these models, in order to follow a routine of financial risk analysis. In our risk modeling process, we try to cover different characteristics of financial time series in a step-by-step way that different aspects of statistical features can be included in measuring the risk.

The structure of this paper is as follows: The second part is the literature review; the third part introduces the relevant model; the fourth part is the data selection and empirical analysis, and finally we draw the conclusion of this paper. 


\section{Introduction to the Theories and Models}

\subsection{PGARCH Model}

Since Engle (1982) proposed the Auto-Regressive Conditional Heteroskedasticity (ARCH) model and used it to analyze the heteroscedasticity of the financial time series, T. Bollerslev (1986) proposed a Generalized Auto-Regressive Conditional Heteroskedasticity (GARCH) model, now GARCH becomes a standard tool for financial econometric analysis. Both two models are able to capture the volatility cluster of assets price changing and the "fat-tail". GARCH model assumes that the yield is subject to the Gaussian distribution, i.e. the yield distribution is symmetrical and hence asymmetric series match with the assumption deficiently. However, many empirical studies show that the yield of assets does not obey the Gaussian distribution strictly, it is an asymmetric skew distribution, and the empirical part of this paper will prove this point.

The PGARCH model improved by the GARCH model can better reflect the asymmetry and the leverage effect. China's futures transaction data meets the general characteristics of financial time series, so we combine Extreme Value Theory and Copula function to measure the VaR and ES under the assumption of Generalized Error Distribution (GED).

The general expression for PGARCH is

$$
\begin{gathered}
y_{t}=x_{t}^{\prime} \phi+\mu_{t}, \mu_{t} \square N\left(0, \sigma_{t}^{2}\right), \\
\sigma_{t}^{h}=\alpha_{0}+\alpha_{1}\left(\left|\mu_{t-1}\right|-\gamma_{1} \mu_{t-1}\right)^{h}+\beta_{1} \sigma_{t-1}^{h},
\end{gathered}
$$

Where $h$ is power value.

\subsection{Extreme Value Theory and Risk Measurement}

Extreme Value Theory was proposed by the Swedish mathematician Nicolas Bernoulli in the eighteenth century. But until the twenties of the last century, EVT came into scholars' vision as a prediction approach. At first, people often used the Gaussian distribution to estimate the possibility of extreme cases, but it may not reflect the real situation well. In 1928, Fisher and Tipper published a paper and eventually introduce EVT into financial timing analysis to fit the tail distribution. The basic content of EVT is consistent with the Gaussian distribution, the greater degree of deviation, the smaller the probability events hold. For describing the tail distribution, fitting approach of EVT is used to cover the statistical characteristics of the yield series.

After decades developing, EVT derives out a large number of related models, which mainly include two types of models, namely BBM model and POT model. The BBM model requires much data in the modeling of the maximum value, while the extreme data is relatively lacking. In practice, BBM model is deficient. However, POT model has a lower requirement for data than the BBM model and it can handle the tail distribution and extreme value well. The POT model based on the General Pareto Distribution only needs to estimate the shape parameter $\xi$ and scale parameter $\beta$. Therefore, in this paper, we use POT model to analyze the tail distribution of the standardized residual series, which is derived from the portfolio yield. The expression of General Pareto Distribution is as follows,

$$
G_{\xi, \beta}(y)=\left\{\begin{array}{cc}
1-(1+\xi y / \beta) & \xi \neq 0 \\
1-\exp (-y / \beta) & \xi=0
\end{array},\right.
$$

Where $\beta>0$ and if $\xi \geq 0$, then $y \geq 0$; if $\xi<0$, then $0 \leq y \leq-\beta / \xi$

According to the contents of the EVT, for a given sufficiently high threshold value $u$, the variable value exceeding the threshold value is classified as the extreme tail and subject to General Pareto Distribution, and its expression can be written as follows,

$$
F(x)=(1-F(u)) G_{\xi, \beta}(x-u)+F(u),(x>u),
$$

Assuming that the number of samples exceeding the threshold value is $N$, replace $F(u)$ with $(n-N) / n$, and use the maximum likelihood method to estimate the GPD parameters $\xi, \beta$, and then we get

$$
\hat{F}(x)=1-\frac{N}{n}\left[1+\frac{\hat{\xi}}{\hat{\beta}}(x-u)\right]^{-1 / \xi},
$$

Firstly, the standardized residual series of yield is sorted in ascending order. Setting the number of extremum as $K$, the threshold value is $Z_{k+1}$, so for $q \geq 1-K / n$, we can get estimated value of the quantile $q$ corresponding to the standardized residual series that is 


$$
\hat{Z}_{q}=Z_{k+1}+\frac{\hat{\xi}}{\hat{\beta}}\left[\left(\frac{1-q}{k-n}\right)^{-\xi}-1\right],
$$

For given $q(q \geq 1-K / n)$, the $\mathrm{VaR}$ of $\mathrm{t}$ to $\mathrm{t}+1$ is estimated as

$$
X_{q}^{t}=\hat{u}_{t+1}+\hat{\sigma}_{t+1} \hat{Z}_{q},
$$

The Expected Shortfall is

$$
E \hat{S}_{q}^{t}=\hat{u}_{t+1}+\hat{\sigma}_{t+1} \hat{Z}_{q}\left[\frac{1}{1-\xi}+\frac{\hat{\beta}-\hat{\xi}_{Z_{k+1}}}{(1-\hat{\xi}) \hat{Z}_{q}}\right],
$$

\subsection{Monte-Carlo Simulation Based on GPD}

Monte-Carlo simulation assumes that the asset price changes follow the form of a random process, we use the computer to complete the simulation process, resulting in a number of possible price path, and to build futures return distribution, and then estimate the VaR and ES in the risk-measurement model. The most commonly used model is Geometric Brownian Motion, the discrete form can be expressed as

$$
\begin{gathered}
\Delta S_{t+i}=S_{t}\left({ }_{t} \Delta t+\delta_{t} \varepsilon \sqrt{\Delta t}\right), \\
S_{t+i}=S_{t}+\Delta S_{t+i},
\end{gathered}
$$

Where $u_{t}$ represents threshold value, given the initial value, it can be calculated by figuring out $S_{t}$; we can get the distribution of random number $\varepsilon$, generated by Copula function.

\section{Empirical Analysis}

\subsection{Sample Selection and Data Processing}

In this paper, we choose the main futures contracts from Chinese commodity futures market; they are five futures contracts of $\mathrm{Au}, \mathrm{Cu}, \mathrm{Al}, \mathrm{Sr}$ and Ta on the Shanghai Futures Exchange. The price data is selected from January 1, 2011, to December 30, 2015, totaling 1230 data points. All of the sample data comes from the Auto-Trader transaction client, and the relevant empirical solution is done using MATLAB and Eviews.

Frist of all, we use the above five futures contracts to build up a portfolio with equal weights and then describe the return on the portfolio. The nature of the bilateral transaction of futures trading makes the realization of asset profit and loss in different ways; we can calculate the logarithmic rate of return by the following formula,

$$
\begin{aligned}
& \ln R_{t}=\ln \left(P_{t}-P_{t-1}\right)-\ln P_{t-1}, \\
& \ln R_{t}=\ln \left(P_{t-1}-P_{t}\right)-\ln P_{t-1},
\end{aligned}
$$

Take the transaction as an example, use the daily closing price of the portfolio to calculate the logarithmic yield series, plot it with MATLAB as shown in Figure 1.

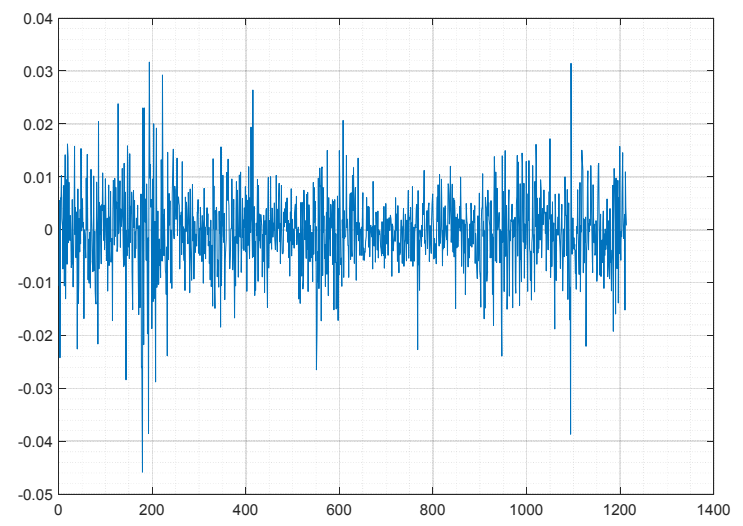

Figure 1. The yield curve of the portfolio

According to the above chart, we can find that the yield series of the portfolio has the following characteristics: the fluctuation of the yield is quite violent, and series has the continuity between the volatility, which may have the volatility cluster effect; the amplitude of the yield is asymmetrical and does not accord with the hypothesis of General Gaussian distribution. 
Through the above analysis, the yield series may not satisfy the characteristics of the symmetric distribution. The autocorrelation test is used for the purpose of further modeling, calculating the logarithmic yield and its square series and finding out the autocorrelation function value of the portfolio, which analyzes whether the distribution of returns is consistent with the independent identically distribution (i.i.d.) hypothesis. The results show that the autocorrelation of the yield series is not significant, and the yield-square series has a large correlation.
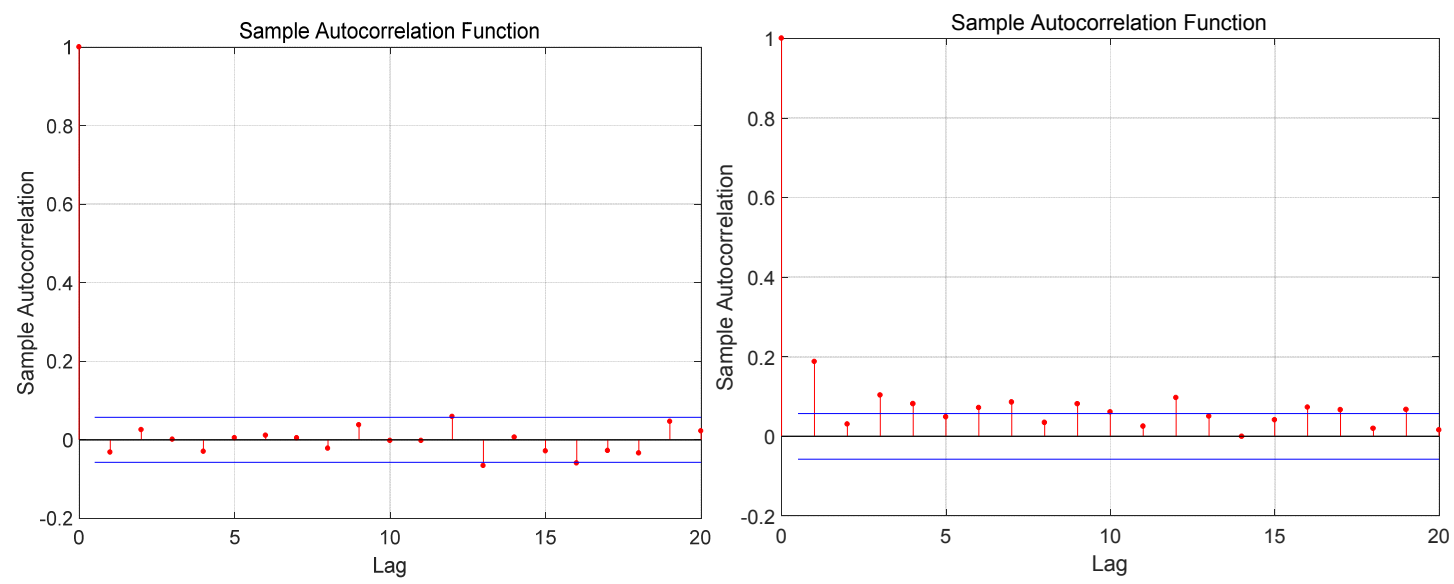

Figure 2. Autocorrelation plot of yield and yield-square series

As can be seen from Figure 2, the ACF of the logarithmic yield series is within two standard deviations. Variables are generally subject to independent identical distribution. The ACF of the yield-square series is larger than the logarithmic yield series, indicating that its autocorrelation is significant and is not suitable for subsequent analysis.

\subsection{Estimation Based on PGARCH-EVT Model}

Through the analysis of the above chapters, the yield series of portfolio satisfies the general characteristics of the financial time series. Assuming that the error is subject to the General Error Distribution, we estimate the conditional variance of the yield series. The samples are analyzed by Eviews, and the conditional mean and sample heteroscedasticity series are separated.

After obtaining the conditional variance and the conditional mean series, it is still necessary to know the distribution of the residual of the samples to calculate the historical VaR and ES values of the portfolio. Standardization of sample residual can be better adapted to subsequent estimates, the formula is as follows

$$
Z_{t}=\frac{x_{t}-u_{t}}{\sigma_{t}}
$$

Where $x_{t}$ is the residual, $u_{t}$ is the conditional mean of the residual; $\sigma_{t}$ is the standardized deviation of residual; $u_{t}$ and $\sigma_{t}$ can be estimated by PGARCH approach.

There are several methods of measuring the standardized residual series, such as parametric method, nonparametric method and semi-parametric. The parametric method that is, given the known distribution, using the samples to solve the parameters of the distribution function, and get the sample distribution. It is usually better to fit the sample distribution at the time of the known general distribution. Due to the atypicality of financial time series, using the known distribution to fit the sample series results in a poor conclusion. The nonparametric method is aimed at estimating CDF, although it is unable to meet the most of the data, especially in the tail fitting. Compared with nonparametric historical simulations and Monte Carlo methods, the Extreme Value Theory in the semi-parametric method can estimate the tail distribution of residual much better.

In the Extreme Value Theory, it is assumed that the sample limit distribution obeys the Generalized Pareto Distribution. First, find the upper and lower limits so that each tail retains a residual of $10 \%$. And then these residual in each tail are reduced to the parameter of GPD below the relevant threshold by the MLE method. Given the excess of each tail, optimize the negative logarithmic likelihood function to estimate the GPD scale parameter $\beta$ and the shape parameter $\xi$.

There are two ways to handle with the residual series. First, retaining the original value of residual, the second is squaring the residual. Risk management needs to control the systematic error, to avoid different conclusion resulted from different data processing approaches. Therefore, we testify the autocorrelation of residual-square and residual series and analyze the degree of independence, as shown in Figure 3. The left side is the residual ACF, the right side of the residual-square of the ACF. 

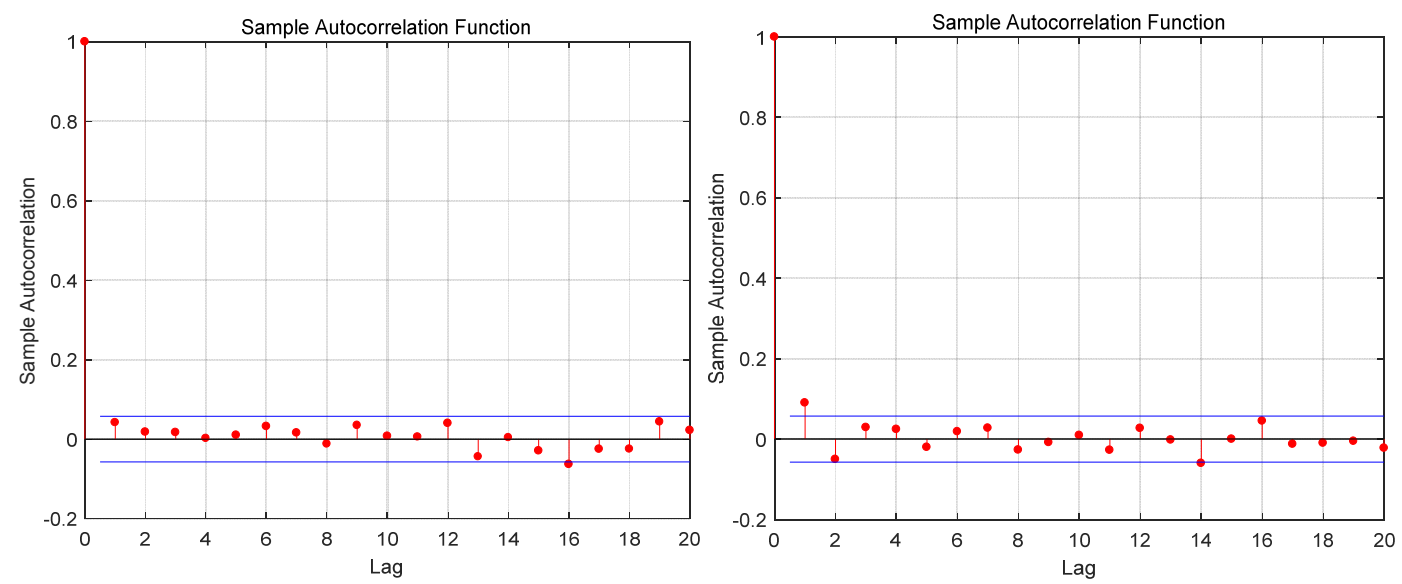

Figure 3. Standardized residual and residual-square of ACF

As shown in Figure 3 that the ACF of residual series is within the two standard deviations, which is consistent with the characteristics of weak sample autocorrelation, and it can be regarded as the prerequisite for the independent identically distribution. Taking the distribution characteristics into account, we use the Generalized Pareto Distribution to model the yield residual series. In modeling the tail distribution, variables are required to obey independent distributions. Therefore, with the support of a large number of sample data, this paper intends to use GPD to fit the distribution of tail data.

In order to further detect the distribution characteristics of the logarithmic yield series, we plot a quantile-quantile plot (Q-Q plot) of the residual series.

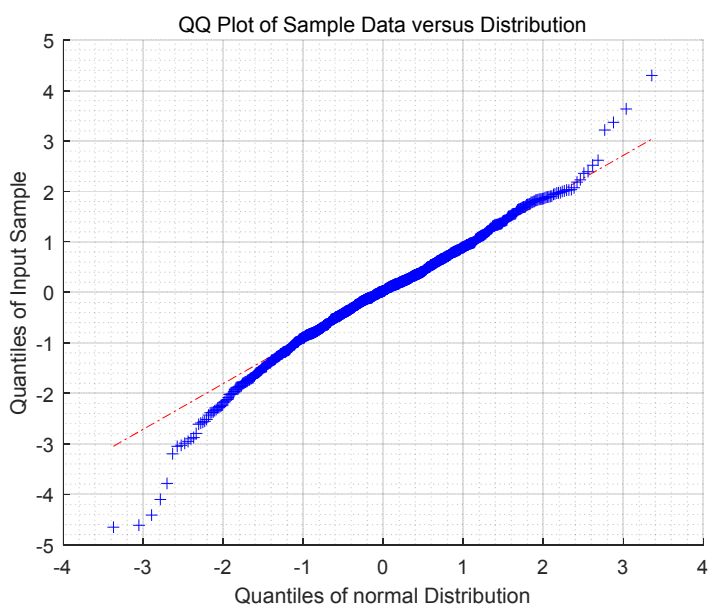

Figure 4. Normal characteristics of portfolio yield distribution

As shown in Figure 4 that the quantile of the sample and the quantile of the Gaussian distribution deviate significantly, which does not meet the assumption of Gaussian distribution, and there exists "fat-tail" characteristic. We can conclude that residual series is much more subject to EVT model.

In this paper, we refer to the two-step method of Danielson and de Vries (2000), which is fitted by the Gaussian kernel estimation method. The upper and lower tail distributions are assumed to be Generalized Pareto Distributions and fitted with the distribution parameters by MLE. The upper and lower tail distribution curves are connected and shown in Figure 5, respectively, in red and blue showing the lower tail and upper tail fitting results. We draw the CDF as shown in Figure 6 below. 


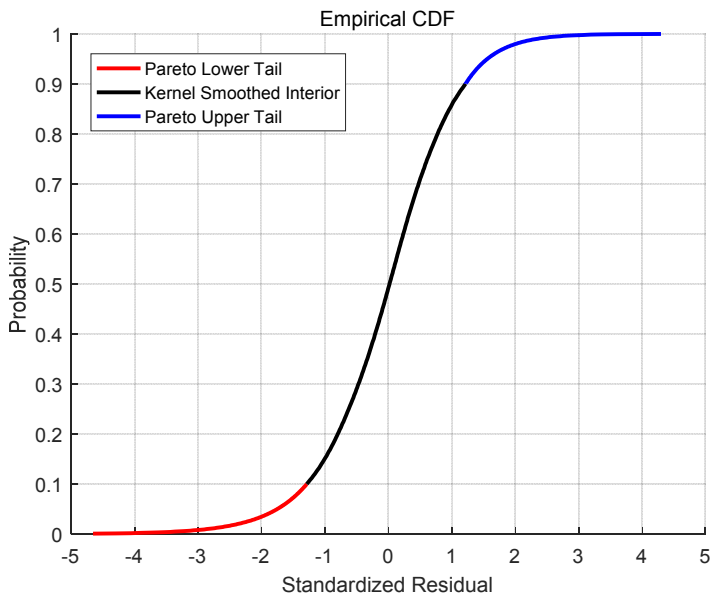

Figure 5. The distribution curve of yield residual

The results of the fitting of the sample CDF need to be further analyzed. The thresholds of 5\% and $10 \%$ are set respectively. We use the K-S test to testify the upper tail fitting effect of the Generalized Pareto Distribution.
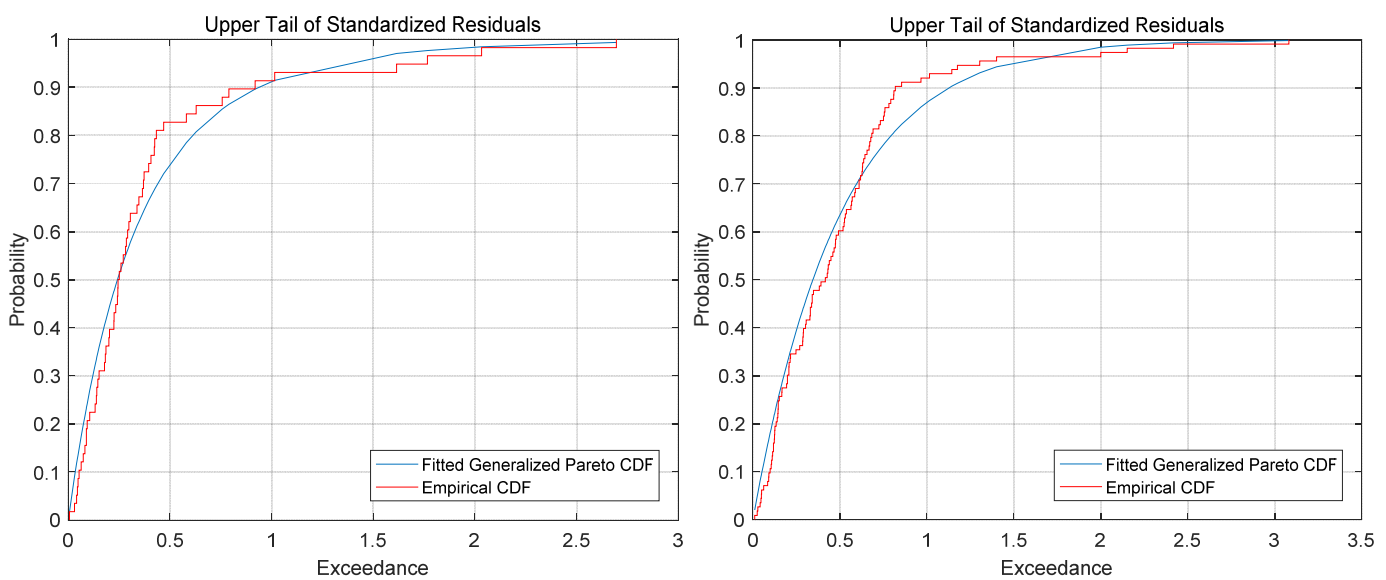

Figure 6. Sample CDF Fitting Effect Analysis (5\% and 10\%)

The results of the K-S test are shown in the following table,

Table 1. K-S test results ( $5 \%$ and $10 \%)$

\begin{tabular}{ccc}
\hline Threshold value & $5 \%$ & $10 \%$ \\
\hline p-value & $5.5543 \mathrm{e}-49$ & $1.0162 \mathrm{e}-100$ \\
Test statistic & 0.9655 & 1 \\
Critical value & 0.1752 & 0.1262
\end{tabular}

Based on the results of Table 1, the upper tail distribution of the fitted residual is approximately identical to the actual results based on the 5\% and 10\% confidence levels and can be used to represent the distribution of the standard residual series.

After determining the distribution of the residual series, we can use Eq. (8) to calculate the risk value and the expected loss of the sample combination from January 1, 2011, to December 30, 2015.

Table 2. The review results: the estimated VaR and ES

\begin{tabular}{ccc}
\hline p-value & $95 \%$ level & $99 \%$ level \\
\hline VaR & 0.0659 & 0.0496 \\
ES & 0.0369 & 0.0309
\end{tabular}

As shown in Table 2, at 95\% confidence level, VaR is unable to cover the data and the estimation is poor to support the risk management. In the actual measurement process, extreme price changes may have a great impact on the results of the calculation; using VaR to measure the asset risk is easy to underestimate the loss caused by unable factors. For the financial supervision departments, it should be paid attention to the hidden danger signal. 


\subsection{Historical Dynamics of VAR and ES Calculations}

The risk analysis of portfolio should also take into account the possibility of fluctuation spillover effect between the assets. The t-Copula function is a good tool to reflect the risk linkage by calculating the correlation coefficient of the assets within the portfolio. We get the degree of freedom is 17.147 and the correlation coefficient matrix $R$ is shown as follows,

$$
R=\left[\begin{array}{lllll}
1 & 0.259143 & 0.617729 & 0.277399 & 0.389372 \\
0.259143 & 1 & 0.350441 & 0.182682 & 0.244291 \\
0.617729 & 0.350441 & 1 & 0.350337 & 0.52846 \\
0.277399 & 0.182682 & 0.350337 & 1 & 0.344031 \\
0.389372 & 0.244291 & 0.52846 & 0.344031 & 1
\end{array}\right],
$$

And then we estimate the maximum possible loss of futures long and short trades, according to CDF of the yield residual series, estimate the VaR and ES of the portfolio during the test period:
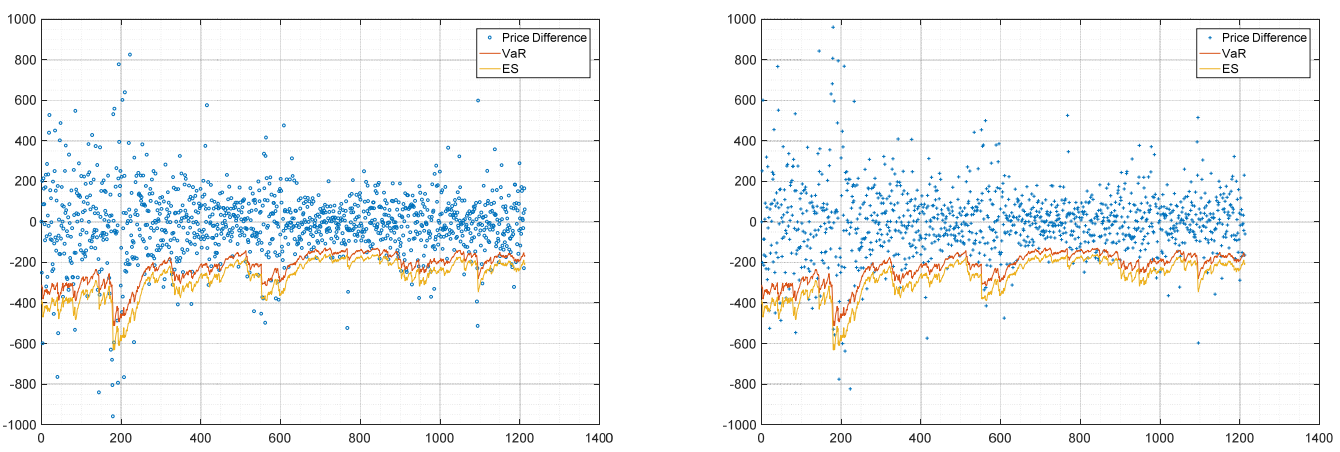

Figure 7. Dynamic VaR and ES estimation (long-left and short-right)

Compared with the estimation effect of VaR and ES, we find that VaR is easily affected by the extreme value at the confidence level of 5\%, which is unsuitable for the risk management. However, ES can well reflect the distribution of yield in the tail and satisfy the basic requirements of risk measurement, it is more suitable for the establishment of risk control index by financial supervision departments.

\subsection{Monte-Carlo Simulation Test}

The risk management of assets not only needs to calculate the VaR based on the historical data but adapts to future uncertainty situation. Therefore, we use the Monte-Carlo simulation method to simulate the future transaction data and estimate the VaR and ES in order to test the reliability of the model.

Using the Monte-Carlo simulation to simulate the future data, we analyze the fitting effect based on the CDF above and compare the VaR and ES.

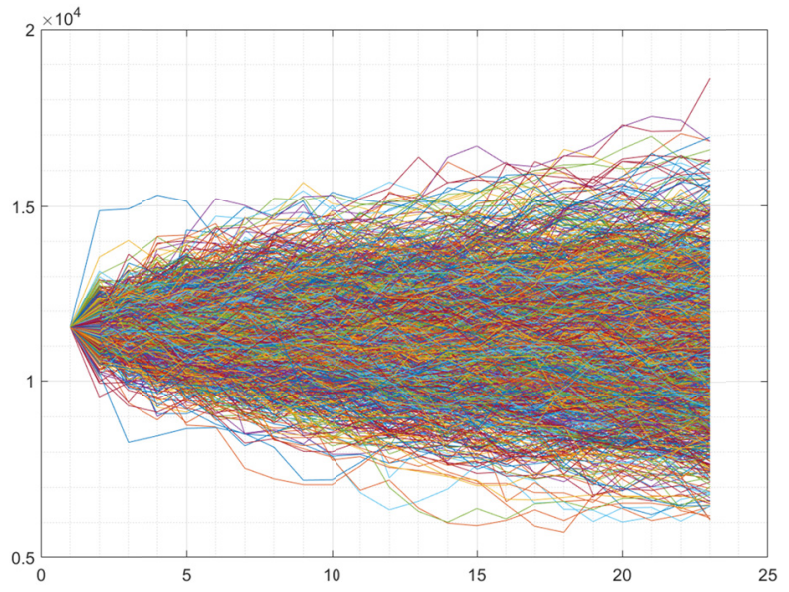

Figure 8. Monte-Carlo simulation path for one-month data 
By Monte-Carlo simulation of futures assets price changes, assuming that the series is subject to General Pareto Distribution, we use the MATLAB to realize the process, and the simulation paths shown in Figure 8. Based on this approach, we estimate the next month, that is, 22 trading days, we find the simulation result as follows,

Table 3. Monte Carlo simulation results

\begin{tabular}{cc|ccc}
\hline \multicolumn{2}{c|}{ Maximum Simulation } & \multicolumn{3}{c}{ Simulated VaR } \\
\hline Gain & Loss & $90 \%$ & $95 \%$ & $99 \%$ \\
$12.6841 \%$ & $9.6616 \%$ & $-5.3358 \%$ & $-6.8495 \%$ & $-10.0796 \%$ \\
\hline
\end{tabular}

Though the analysis of the above Table 3, we can conclude that the maximum expected loss of futures market can be controlled with $6 \%$ under the confidence level of $90 \%$, and the overall risk can be controlled under different confidence levels. Financial supervision departments can establish different standards of confidence level based on different market conditions, so as to adjust the threshold of asset price changes in order to control the risk level of the capital market transaction.

\section{Conclusion and Policy Suggestion}

Financial risk management is an important part of financial supervision. We take the sample of Shanghai Futures market as an example, and then we calculate the risk value and expected loss of portfolio, and finally conclude that the risk is controllable at a certain level of confidence. In this paper, according to the basic process of financial modeling, we compared with the characteristics of different models and choose the most appropriate model for measuring financial risk and expected loss, the results show that our model can reflect the financial risk of portfolio and assets in both historical and simulated data very well. The risk measurement based on PGARCH-EVT-Copula model can fully reflect the asymmetric distribution of financial time and measure the fluctuation spillover effect between assets. Each sub-model in this paper is combined with their characteristics as follows,

- The PGARCH model is an improved asymmetric model based on GARCH model, which reflect the asymmetric characteristics of financial time series. In this paper, the residual-square series divided from PGARCH model is close to the real random fluctuation and better for risk management. There is less systematic error;

- EVT model as a semi-parametric method does not require more data support. We get rid of the limitations of parameter estimation. EVT analyzes the upper and lower tail distribution of financial time series and reflects the abnormal fluctuations in financial asset prices changes, fitting by the General Pareto Distribution. To some extent, we will be more close to the real changes of extreme value;

- Copula theory is used to reflect the fluctuation spillover effect of assets by calculating the correlation coefficient of each asset in the portfolio. The yield series of assets in the portfolio often shows autocorrelation, such as the metal futures contracts used in this paper. It can be calculated in Copula function that the correlation coefficient of the assets, so as to obtain the quantitative description of correlation of different assets.

Risk management requires good indicators to reflect the level of asset risk. This paper compares VaR and ES's effects in measuring risk and finds that the ES proposed on the basis of VARs can better respond to the extreme situation of loss and give the possibility of extreme loss. In this paper, five futures contracts in China Shanghai futures market are built a financial portfolio. By calculating the ES of the portfolio, we conclude that at a certain probability level, when the market risk is less than the threshold value, the overall risk is in the state of control; when the market risk exceeds the threshold value. It is acceptable in some probability, but if the risk of overloading continues, it may indicate that the risk is gradually widening, and the possibility of financial crisis is increasing. It is necessary for financial supervision departments to take appropriate action and adjust the market state, controlling the risk at a reasonable level.

Financial supervision departments should be wary of the financial crisis signals. In order to evade the future risk uncertainty, building up financial control model with historical data to reflect the trend of risk management has reference significance.

\section{Reference}

Acerbi, C., Nordio, C., \& Sirtori, C. (2001). Expected shortfall as a tool for financial risk management. arXiv preprint cond-mat/0102304.

Andersen, T., Bollerslev, T., Diebold, F., \& Ebens, H. (2000). The Distribution of Stock Return Volatility. https://doi.org/10.3386/w7933

Andreev, V. O., Tinyakov, S. E., Parahin, G. P., \& Ovchinnikova, O. P. (n.d.). An Application of EVT, GPD and POT Methods in the Russian Stock Market (RTS Index). SSRN Electronic Journal. https://doi.org/10.2139/ssrn.1507678

Ayusuk, A., \& Sriboonchitta, S. (2014). Risk Analysis in Asian Emerging Markets using Canonical Vine Copula and 
Extreme Value Theory. Thai Journal of Mathematics, 59-72.

Binder, K., \& Heermann, D. W. (1992). Theoretical Foundations of the Monte Carlo Method and Its Applications in Statistical Physics. Monte Carlo Simulation in Statistical Physics, 5-67. https://doi.org/10.1007/978-3-662-30273-6_2

Bollerslev, T. (1986). Generalized autoregressive conditional heteroskedasticity. Journal of Econometrics, 31(3), 307327. https://doi.org/10.1016/0304-4076(86)90063-1

Clayton, D., \& Cuzick, J. (1985). Multivariate Generalizations of the Proportional Hazards Model. Journal of the Royal Statistical Society. Series A (General), 148(2), 82. https://doi.org/10.2307/2981943

Demarta, S., \& McNeil, A. J. (2007). The t Copula and Related Copulas. International Statistical Review, 73(1), 111-129. https://doi.org/10.1111/j.1751-5823.2005.tb00254.x

Embrechts, P., Resnick, S. I., \& Samorodnitsky, G. (1999). Extreme Value Theory as a risk management tool. North American Actuarial Journal, 3(2), 30-41. https://doi.org/10.1080/10920277.1999.10595797

Engle, R. F. (1982). Autoregressive Conditional Heteroscedasticity with Estimates of the Variance of United Kingdom Inflation. Econometrica, 50(4), 987. https://doi.org/10.2307/1912773

Glosten, L. R., Jagannathan, R., \& Runkle, D. E. (1993). On the Relation between the Expected Value and the Volatility of the Nominal Excess Return on Stocks. The Journal of Finance, 48(5), 1779. https://doi.org/10.2307/2329067

Hansson, S. O. (2001). A Descriptive Framework For Public Risk Management. Risk Management, 3(3), 23-32. https://doi.org/10.1057/palgrave.rm.8240091

Jalal, A., \& Rockinger, M. (2008). Predicting tail-related risk measures: The consequences of using GARCH filters for non-GARCH data. Journal of Empirical Finance, 15(5), 868-877. https://doi.org/10.1016/j.jempfin.2008.02.004

Ling, S., \& McAleer, M. (2002). Necessary and sufficient moment conditions for the GARCH (r, s) and asymmetric power GARCH (r, s) models. Econometric Theory, 18(3), 722-729. https://doi.org/10.1017/S0266466602183071

Scarrott, C. (2015). Univariate Extreme Value Mixture Modeling. Extreme Value Modeling and Risk Analysis, 41-67. https://doi.org/10.1201/b19721-4

Simar, L. (1992). Estimating Efficiencies from Frontier Models with Panel Data: A Comparison of Parametric, Non-Parametric and Semi-Parametric Methods with Bootstrapping. International Applications of Productivity and Efficiency Analysis, 167-199. https://doi.org/10.1007/978-94-017-1923-0_11

Tully, E., \& Lucey, B. M. (2007). A power GARCH examination of the gold market. Research in International Business and Finance, 21(2), 316-325. https://doi.org/10.1016/j.ribaf.2006.07.001

Verhoeven, P., \& McAleer, M. (2004). Fat tails and asymmetry in financial volatility models. Mathematics and Computers in Simulation, 64(3-4), 351-361. https://doi.org/10.1016/S0378-4754(03)00101-0

Wang, J. S. (2002). Efficient Monte Carlo Simulation Methods in Statistical Physics. Monte Carlo and Quasi-Monte Carlo Methods 2000, 141-157. https://doi.org/10.1007/978-3-642-56046-0_9

Wang, Z. R., Chen, X. H., Jin, Y. B., \& Zhou, Y. J. (2010). Estimating the risk of foreign exchange portfolio: Using VaR and CVaR based on GARCH-EVT-Copula model. Physica A: Statistical Mechanics and Its Applications, 389(21), 4918-4928. https://doi.org/10.1016/j.physa.2010.07.012

Yang, J., Yang, Z., \& Zhou, Y. (2011). Intraday price discovery and volatility transmission in the stock index and stock index futures markets: Evidence from China. Journal of Futures Markets, 32(2), 99-121. https://doi.org/10.1002/fut.20514

\section{Copyrights}

Copyright for this article is retained by the author(s), with first publication rights granted to the journal.

This is an open-access article distributed under the terms and conditions of the Creative Commons Attribution license which permits unrestricted use, distribution, and reproduction in any medium, provided the original work is properly cited. 